\section{Signalling a change}

We all fancy a new look from time to time, but during their development many organisms can take this one step further and undergo momentous changes in shape - a phenomenon that is exemplified by insect metamorphosis. In their study in Developmental Cell, Martín-Blanco and colleagues have shed new light on some of the basic steps that underlie these processes.

In Drosophila melanogaster, the exoskeleton of the adult (or imago) develops from epithelial structures that grow inside the larval stages of the fly. These structures are known as imaginal discs and are attached to the larval epithelium by a thin stalk of cells. They contain columnar cells, which form the imaginal epithelium, and squamous cells, which make up the periodial epithelium and stalk (PS). During development, the imaginal discs move to the outside of the larval epidermis in a process known as disc eversion.

Previously, changes in cell shape were thought to be sufficient for eversion - causing the disc to move through the widened disc stalk to the outside of the larval epidermis. But

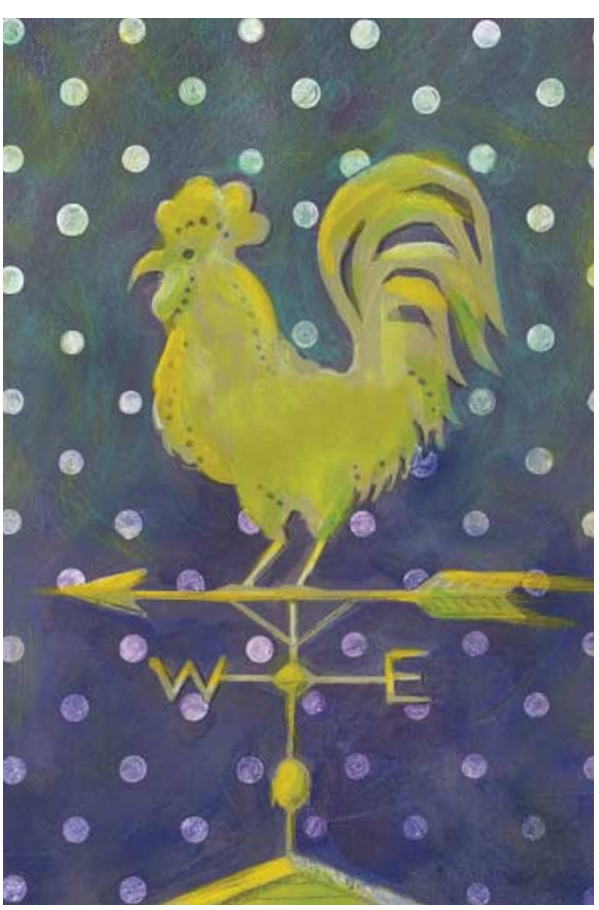

now, Martín-Blanco and colleagues present a new model for imaginal disc eversion that is based on their in vivo observations of imaginal morphogenesis and their detailed study of histological sections.

They found that, initially, a positional change occurs such that the PS cells of the discs become apposed to the larval epidermis. The PS cells then undergo a pseudo-epithelial-mesenchymal transition (PEMT). During PEMT, they lose their basal membrane, and proteins from the zonula adherens and septate junctions are redistributed from the cell membrane to the cytoskeleton - thereby disrupting cell adhesion. Marked cytoskeletal reorganization occurs and the cells become motile and invade the larval epithelium. The loss of adhesion among these invading cells generates perforations in the epithelium, which coalesce with each other and the disc stalk to produce a single hole. The continued intercalation of PS cells at the edges of the hole further widens it, which allows the extrusion of the imaginal disc and leaves a stripe of PS cells surrounding the disc. These cells then make up the leading front of the disc and guide its expansion and migration across the larval epithelium.

The Jun N-terminal kinase (JNK) signalling pathway — which includes the proteins Hemipterous (the JNKkinase homologue), Basket (the JNK homologue) and Puckered (a phosphatase that inactivates Basket) in D. melanogaster - was found to have a significant role in driving many aspects of disc eversion.

Together, these findings have produced a model for epithelial perforation that might well provide insight into analogous epithelial-invasion processes that occur in the development of other organisms.

Lesley Cunliffe

(1) References and links ORIGINAL RESEARCH PAPER

Pastor-Pareja, J. C. et al. Invasive cell behaviour during Drosophila imaginal disc eversion is mediated by the JNK signaling cascade. Dev. Cell 7, 387-399 (2004)

\section{IN BRIEF}

\section{CELL MIGRATION}

Two distinct actin networks drive the protrusion of migrating cells.

Ponti, A. et al. Science 305, 1782-1786 (2004)

Cell migration requires plasma-membrane protrusion, which is brought about by actin polymerization at the leading edge of the cell. Using quantitative fluorescent speckle microscopy, Ponti et al. were able to distinguish between two actin networks that colocalized at the leading edge of epithelial cells. A lamellipodium network promoted random protrusion and retraction, whereas the lamella caused productive cell advance, thanks to the coupling of actomyosin contractile forces to substrate adhesion.

\section{DEVELOPMENTAL CELL BIOLOGY}

\section{Mesenchymal-epithelial transition during somitic} segmentation is regulated by differential roles of Cdc42 and Rac1.

Nakaya, Y. et al. Dev. Cell 7, 425-438 (2004)

The authors studied the effects of different levels of Racl and Cdc42 activity on the transition between mesenchymal and epithelial states of cells during somitogenesis in chick embryos. The level of Cdc42 activity was important for the decision between epithelial and mesenchymal cell states. Specific levels of Racl activity were necessary for correct epithelialization.

\section{CELL GROWTH}

\section{A dynamic transcriptional network communicates growth} potential to ribosome synthesis and critical cell size.

Jorgensen, P. et al. Genes Dev. 1 Oct 2004 (doi:10.1101/gad.1228804)

Cells need to reach a critical size before they commit to cell division (a point called Start in budding yeast). The authors identified two Start repressors — Sfp 1 and Sch9 — that are also activators of a transcriptional network that regulates ribosome biogenesis. The abundance of Sch9 and the nuclear localization of Sfp 1 were regulated by nutrient status. In turn, Sfp 1 regulates ribosome synthesis in response to nutrients. So, nutrient status determines the critical cell-size threshold by Sfp1- and Sch9-mediated control of ribosome synthesis.

\section{CHROMATIN}

Human SirT1 interacts with histone $\mathrm{H} 1$ and promotes formation of facultative heterochromatin.

Vaquero, A. et al. Mol. Cell 16, 93-105 (2004)

Vaquero and colleagues identified SirT1 as a human histone deacetylase with a preference for histone H4 lysine 16 (H4-K16), and showed that SirT1 interacts with, and deacetylates, H1-K26. SirT1-mediated gene silencing seems to involve a functional role for $\mathrm{H} 1$ - expression of a SirT1 reporter resulted in the deacetylation of $\mathrm{H} 4-\mathrm{K} 16$, the recruitment of $\mathrm{H} 1$ to the promoter and the subsequent loss of H3-K79 methylation, which is characteristic of heterochromatin. 